\title{
Neuer Therapiestandard bei juveniler Dermatomyositis?
}

\author{
In der Therapie der juvenilen Dermatomyositis wird sich möglicherweise \\ ein neuer Standard etablieren. In einer randomisierten Studie war die \\ Glukokortikoid-Monotherapie, bisher erste Wahl, einer Kombination mit \\ Immunsuppressiva unterlegen.
}

Z ur medikamentösen Behandlung der juvenilen Dermatomyositis mangelte es bislang an kontrollierten Studien. Nun liegt eine internationale Multicenter-Studie vor, die in drei Armen die Regime Prednison allein, Prednison plus Ciclosporin sowie Prednison plus Methotrexat vergleicht.

An der Studie waren 139 Kinder und Jugendliche im Alter von höchstens 18 Jahren beteiligt. Diese erhielten zunächst eine Pulstherapie mit Methylprednisolon $(3 \times$ tägl. $30 \mathrm{mg} / \mathrm{kg}$ i.v.); danach wurden sie einer der drei Gruppen zugelost. Ciclosporin wurde oral in Dosen von 4-5 $\mathrm{mg} / \mathrm{kg}$ pro Tag über mindestens zwei Jahre verabreicht. Methotrexat erhielten die entsprechenden Teilnehmer einmal wöchentlich s.c. oder i.m. in einer Dosierung von 15-20 $\mathrm{mg} / \mathrm{m}^{2}$ über ebenfalls mindestens zwei Jahre. Das Kortikosteroid wurde in einer zweimonatigen Induktionsphase von anfänglich $2 \mathrm{mg} / \mathrm{kg}$ schrittweise auf $1 \mathrm{mg} / \mathrm{kg}$ - verteilt auf drei Tagesdosen - titriert. Danach erfolgten weitere Dosisreduktionen bis auf $0,1 \mathrm{mg} / \mathrm{kg}$, die nach insgesamt zwei Jahren nur noch jeden zweiten Tag gegeben wurden. Wenn die Erkrankung bis zu diesem Zeitpunkt in ein inaktives Stadium eingetreten war, konnte Prednison abgesetzt werden.

Sechs Monate nach der Randomisierung hatten $51 \%$ der Patienten unter Prednison, $70 \%$ unter Prednison plus Ciclosporin und $72 \%$ unter Prednison plus Methotrexat eine signifikante Verbesserung ihres Krankheitsbilds erzielt. Als Maßstab galt eine Verbesserung im PRINTO-Score um mindestens $20 \%$ bei drei oder mehr von insgesamt sechs Variablen, wobei sich höchstens eine Variable um mehr als $30 \%$ verschlechtert haben durfte. Diese Variablen waren: Muskelstärke, Krankheitsaktivität aus Sicht des Arztes sowie nach dem
Disease Activity Score, Funktionalität nach C-HAQ, allgemeines Wohlbefinden aus Sicht der Eltern und gesundheitsbezogene Lebensqualität.

Die Teilnehmer wurden im Mittel 35,5 Monate nachbeobachtet. In dieser Zeit kam es bei acht, sieben beziehungsweise 15 Patienten zu einer Remission. Damit lag die Remissionsrate in der Methotrexat-Gruppe im Vergleich zu den beiden anderen um das 2,45-Fache höher. Erstere hatte zudem deutlich schneller ein inaktives Stadium erreicht ( $p=0,021)$. Zu einem Therapieversagen, definiert als Notwendigkeit einer $\mathrm{Zu}$ satztherapie oder als Therapieabbruch, kam es bei 30 Patienten unter Monotherapie sowie bei 20 und 17 Patienten in den anderen beiden Gruppen. Nebenwirkungen, vor allem Hypertrichose, Hirsutismus oder Alopezie, traten unter Ciclosporin deutlich vermehrt auf. Bis zum Absetzen des Steroids dauerte es in der Monotherapiegruppe im Mittel 35,8 Monate. In den beiden anderen Gruppen ging dies deutlich schneller (29,4 bzw. 29,7 Monate). Letztlich konnten 19,31 respektive 25 Patienten auf Prednison verzichten.

Fazit: Wie die randomisierte Studie zeigt, ist die Kombination mit einem Immunsuppressivum der Glukokortikoid-Monotherapie an Effektivität deutlich überlegen. Dabei sprechen sowohl das Sicherheitsprofil als auch der steroidsparende Effekt für die Kombination mit Methotrexat. Letztere hat den Autoren zufolge das Potenzial, neuer Behandlungsstandard in der Therapie der juvenilen Dermatomyositis zu werden.

Dr. Elke Oberhofer
Ruperto $\mathrm{N}$ et al. Prednisone versus prednisone plus ciclosporin versus prednisone plus methotrexate in new-onset juvenile dermatomyositis: a randomised trial. Lancet $2016 ; 387$ : 671-8 